\title{
Radioactive Target Detection Using Statistical Method
}

\author{
Sherief Hashima \\ Engineering Dept, Nuclear Research Center, Egyptian Atomic Energy Authority, Inshas, Egypt \\ ng_shrif@yahoo.com
}

\begin{abstract}
Radioactive materials are extremely dangerous as it threatens the earth survival. They contaminate the environment with a lot of damages. Nowadays revealing radioactive material become an important topic for public health, national security, and environmental services. This paper discusses a statistical method to detect and identify an unobserved radioactive source, where the counts of radiation particles received by those detectors are spatially distributed of Poisson distribution. Also, each count composed of a signal and a background. This paper provides a numerical method to estimate the strength and location of unobserved radioactive source based on the physical law of signal degradation with distance. Based on these estimates, a likelihood ratio statistic is used to test the existence of the source. Simulation results show that the proposed statistical method is efficient for revealing the unobserved radioactive source.
\end{abstract}

Keywords: Radiation and Radioactive Material, Spatial Statistical Method, Poisson distribution.

\section{INTRODUCTION}

Radioactive materials, also known as radioactive isotopes or radioisotopes, have unique atoms with different numbers of protons or neutrons from ordinary atoms. These specific atoms include nuclei that decay and emit alpha, beta particles, or gamma rays until the isotope comes stable. Radioactive materials have safely benefited the society in a lot of applications, including X-rays, Magnetic resonance image (MRI), and nuclear energy. Nevertheless, these materials can also be harmful to the public audience. Here we provide some real examples of nuclear accidents that occurred around the world: -

Example 1 (Fukushima disaster)The Fukushima Daiichi nuclear disaster caused by the earthquake in Japan on March 11, 20011 has made severe nuclear pollution in the world. The earthquake caused a 14-meter-high tsunami that swept over the plant's seawall (12 m height) and flooded the plant's lower grounds around the Units 1-4 reactor constructions with seawater, filling the bunkers and hitting the emergency generators. Large amounts of water polluted with radioactive isotopes were released into the Pacific ocean during and after the disaster. Up till now, there still severe outcomes of the nuclear crisis that continues to Japan. Although the main cause was from the nature, this accident appears a huge safety and security problems that exist in all of the nuclear power plants (NPPs) all over the world. In NPPs, large amounts of nuclear fuel and waste that contain uranium or other radioactive materials exists. In order to improve the safety and security level of a NPP, it is important to develop a fast and accurate detection system for the nuclear unit's leakage.

Example 2 (Radon detection) Radon is a colorless, unperfumed, and tasteless radioactive gas produced during the natural decay of radium, uranium, and thoron in soil, rock, and water. Its relationships with various diseases, particularly lung cancer, are well known. Radon can penetrate homes through cracks and holes. Also, it might contaminate the well water. Although ease of detection of radon level nowadays in residence, ways to determine the source of decay have not been wellstudied. Detection and further removal of the decay source can lower the radiation pollution generated by radon and accordingly improve the environments of nearby residents [1].

Example 3 Recently several countries like USA and UK have acknowledged the high possibility of terrorist attacks using weapons of mass destruction (WMD) [2]. A good summary of physical models of the nuclear weapons is surveyed in Fetter et al. [3]. Because a hidden nuclear weapon contains radioactive materials, which emit radiation from its surface, methods of radioactive detection should be improved especially in countries that face nuclear threats.

Example 4 (Nuclear waste leakage detection) the nuclear power industry and also industrial petroleum digging companies create thousands of tons of deadly nuclear wastes every year all over the world [4]. These wastes need to be transported to special treatment centers, which can impose potential damage to the health and economic well-being of residents living on and near the route [5].

Additionally, nuclear fuel and waste contain uranium, or other radioactive materials are utilized in all NPPs. They are sealed in fuel rods that are bundled together into 
nuclear fuel associations. If these materials leak from the rods, millions of people will be put at risk over many years because leaking waste can move past homes, workplaces, recreational areas, schools, and hospitals. A person standing but one yard from an unshielded, 10year-old fuel assembly would receive a fatal dose of radiation in a few three minutes. A thirty-second exposure at the same space would significantly increase the risk of cancer and genetic damage. Therefore, it is critical to quickly and accurately detect and locate any leakage of nuclear waste along the transportation routes of these materials for efficient damage control.

The rest of the paper is organized as follow, Section 2 discusses the related work. Section 3 previews the proposed statistical method. Section 4 previews the simulation results of the proposed method. Finally, Section 5 concludes the work.

\section{RELATED WORK}

The authors in [6] created an early warning and alert system for radioactive material radiation leakage based on wireless sensor networks. The system was done by implementing standard web service interfaces like sensor alert and web notification services. A system based on WSNs to monitor environmental conditions around and inside a NPP, specifically, radiation levels. Sensor nodes, equipped with radiation sensors, are deployed in fixed positions in [7]. However, this system uses fixed sensor nodes and in nuclear sites only. The authors in [8] evaluated algorithms that integrate methods of parametric and Bayesian statistics for radiation detection and tracking in large cities. The results of analyses and simulations are compared against measurements made on a reduced scale testbed. They also surveyed the background radiation in the city of Sacramento is used to quantify the efficacy of police patrols to detect threats. The problem of estimating the location of a target on a plane using random measurements of sensors' distancedifferences to the target is discussed in [9]. They presented a simulation-based comparison of two localization methods based on mean of estimators and means of measurements, respectively. They also proposed a method for localizing point radiation sources from intensity measurements which uses the localization as a component. However, in order to get accurate results, the number of sensors and estimators should increase. In [10] the authors addressed the use of multiple detectors, mounted on moving vehicles, for detecting nuclear threats. An example scenario is that of multiple taxi cabs each carrying a detector with known position using GPS. The level of detected risk is reported from each detector at each position. The authors modeled and formulated the problem of using spatially deployed mobile detector networks to identify and locate risks. They also considered the false-positive and false negative reports from individual sensors in their simulations.

\section{STATISTICAL METHOD}

Based on the physical law discussed in Fetter et al. (1990), the radiation rate decreases inversely with the square of the distance between the source and the detector, and the number of radiation count received by the detector follows a homogeneous Poisson process with intensity rate per time unit equal to $\mathrm{Aev}_{\mathrm{s}} /(4 \pi \mathrm{r} 2)$ where $\mathrm{r}$ is the distance between the radioactive source and the detector. $\mathrm{e}$ and $\mathrm{A}$ are the efficiency and area of the detector. vs is the surface radiation rate per time unit of the radioactive source. The total number of counts, the detector receives form the source follows poison distribution with expected value as

\begin{tabular}{|c|c|}
\hline$\frac{T A e v_{s}}{4 \pi r^{2}}$, & (1) \\
\hline
\end{tabular}

There is also natural background that the detector should put into its account. Assume that the background and detected signal are independent. The background radiation follows Poison distribution with intensity rate per unit time equal to

$$
A e v_{b}
$$

Where $v_{b}$ is the background intensity rate per area unit per unit time. If the detection process consumes time $\mathrm{T}$, then the count of background radiation follows Poison distribution with value equal to

\section{$\mathrm{TAev}_{b}$}

Hence, the total number of counts received by the detector is the combination of the two Poisson distributions given in eqs $(1,2)$. Since the sum of two poison distribution is another poison distribution, the total number of counts per detector follows Poisson distribution with a value

$$
\begin{array}{|r|r|}
\hline \frac{T A e v_{s}}{4 \pi r^{2}}+T A e v_{b} & \\
=T A e\left(\frac{v_{s}}{4 \pi r^{2}}+v_{b}\right) & \\
\hline
\end{array}
$$

The signal intensity rate $v \mathrm{~s}$ is completely determined by the radioactive source and is typically unknown. The background intensity rate $v_{\mathrm{b}}$ may be known in some cases if there are independent experiments to measure it; otherwise it must also be treated as an unknown

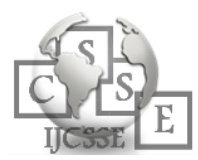


parameter. Moreover, because the location of the radioactive source is unknown, the distance $r$ involved in (3) is also unknown.

We use the bootstrap method to compute the probability of detection p. The steps are as follow:

(1) Compute the observed value based on the observed counts y1,....ym.

(2) Estimate the counts

(3) Generate random variables with total count numbers

(4) Use bootstrap method to estimate the $p$ value.

According to the resulted $\mathrm{P}$ value, We can define the detection of existing radioactive source or not.

\section{SIMULATION RESULTS}

We assumed the existence of a hidden radioactive source if the p-value was less than 0.05. The simulation was repeated for 1000 times for each selected value of $s_{0}$. The power function at each $s_{0}$ value was assessed by the rejection rate of significance for

The estimation accuracy of the location of the radioactive source was assessed for all the sigificance results by the mean square errors of the Euclidean distance between the estimated and the designated point locations. Specifically, we define the "Mean Distance" as

$$
\begin{aligned}
& \text { Mean Distance } \\
& =\frac{\sum_{k=1}^{1000}\left\|\hat{a}_{0_{k}}-a_{0}\right\| I_{p_{k}}<0.05}{\sum_{k=1}^{1000} I_{p_{k}}<0.05}
\end{aligned}
$$

where $\mathrm{p}_{\mathrm{k}}$ is the bootstrap p-value of the kth replication in the simulation for selected $\mathrm{s}_{0}$

value. A smaller MD indicates an accurate localization of the radioactive source.

Figure 1 displays the power function of with selected values of $\mathrm{s}_{0}$. The type I error probability was displayed by the case in which s $0=0$. As we expected, for the proposed method, the power function was close to 0.05 when s 0 approached 0 . When s 0 was less than or equal to 2.5 , the power function was less than 0.1 . As s 0 became stronger, the power function increased rapidly to almost 1 .

Figure 1 displays the results of the location specification for our proposed method and

Kulldorff's scan method. It can be seen from the figure that the MD decreased as $s_{0}$ increased. However in Kulldorff's scan method, the MD value did not converge to 0 as $\mathrm{s} 0$ goes to $\infty$. This is because the collection of cluster candidate's C is finite in Kulldorff's scan method.

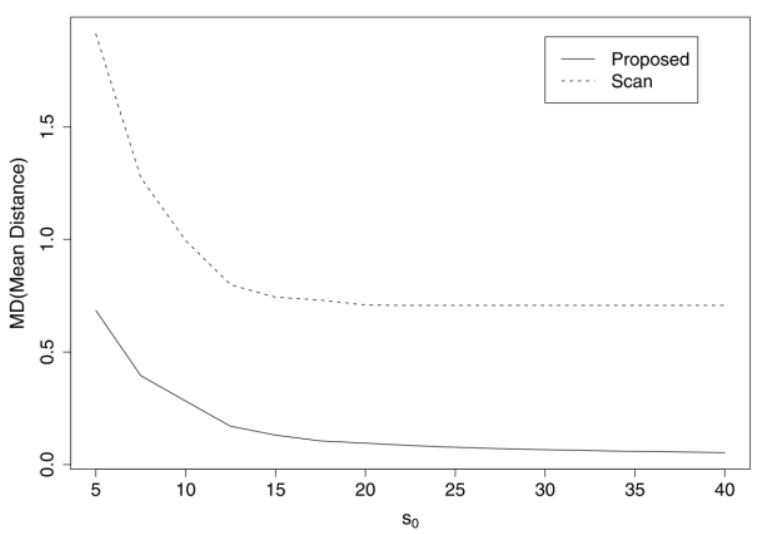

Fig. 1. Mean distances (MDs) of location for proposed and scanned methods

Note that our simulation results displayed in Figure 1 represent a broad class of cases in real applications. More specifically, results of the simulation can be extended to cases that have not been displayed. A hidden radioactive source can be successfully detected and localized by carefully allocating detectors. Further simulation studies (not shown) also suggested that the results of the detection and localization were not sensitive to the location of the source as long as it was not near the boundary or outside the network.

\section{CONCLUSION}

In this paper, we have proposed a statistical method as well as the algorithm for nuclear radiation target detection. Comparing with methods using single detectors, our method can simultaneously detect and locate nuclear radioactive targets. Numerical results showed that our algorithm could successfully fulfill our tasks. The idea presented by this paper will be extensively used to real world nuclear safety and security problems.

\section{REFERENCES}

[1] Dersch, R. (2004). Primary and secondary measurements of 222Rn. Applied Radiation and Isotopes, 60, 387-390.

[2] Richelson J (2002) Defending nuclear terror. Bull At Sci 58:38-43

[3] Fetter S, Frolov VA, Miller M, Mozley R, Prilutsky QF, Rodionov SN, Sagdeev RZ (1990) Detection nuclear warheads. Sci Glob Secur 1:225-302

[4] Drew, C. H., Grace, D. A., Silbernagel, S. M., Hemmings, E. S., Smith, A., Griffith, W. C., Takaro, T. K., \& Faustman, E. M. (2003). Nuclear waste transportation: case studies of identifying stakeholder risk information needs. Environmental Health Perspective, 111, 263-272. 
[5] Gawande, K., \& Smith, H. (2001). Nuclear waste transport and residential property values: estimating the effects of perceived risks. Journal of Environmental Economics and Management, 42, 207-233.

[6] S. Shafi, A. reshi, and A. Kumaravel," Wireless Sensor Network Based Early Warning and Alert System for Radioactive Radiation Leakage", Middle-East Journal of Scientific Research 19 (12): 1602-1608, 2014.

[7] Barbarán, Javier \& Díaz, Manuel \& Esteve, Iñaki \& Rubio, Bartolomé. (2007). RadMote: a mobile framework for radiation monitoring in nuclear power plants.

[8] A. H. Liu, J. J. Bunn and K. M. Chandy, "Sensor networks for the detection and tracking of radiation and other threats in cities," Proceedings of the 10th ACM/IEEE International Conference on Information Processing in Sensor Networks, , pp. 1-12,Chicago, IL, 2011.

[9] N. S. V. Rao et al., "Localization under random measurements with application to radiation sources," 2008 11th International Conference on Information Fusion, Cologne, pp. 1-8, 2008.

[10] Hochbaum, D.S. \& Fishbain, B. Ann Oper Res, "Nuclear threat detection with mobile distributed sensor networks", Annals of Operations Research, pp 45-63 (2011).

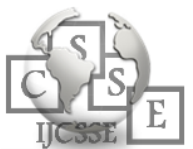

